\title{
Ceguera y trastornos neurológicos por monóxido de carbono: tratamiento efectivo con oxígeno hiperbárico en dos pacientes pediátricos
}

\author{
Blindness and neurological sequelae following carbon monoxide poisoning: \\ effective treatment with hyperbaric oxygen
}

Dra. Patricia C. Cardoso ${ }^{a}$ Dra. Alicia La Fuente ${ }^{b}$, Dra. Nina Subbotina ${ }^{c}$ y Dra. Elda G. Cargnel ${ }^{a}$

\section{RESUMEN}

Se presentan dos pacientes que desarrollaron deterioro visual debido a una intoxicación por monóxido de carbono. Ellos fueron tratados con oxígeno hiperbárico y recuperaron no solo su visión, sino que, además, mejoraron su signo-sintomatología neurológica. Se cree que la implementación de oxígeno hiperbárico, incluso en un período tardío, será efectiva para revertir las secuelas neurológicas.

Palabras clave: intoxicación por monóxido de carbono, ceguera, lesiones encefálicas, niño, oxigenación hiperbárica.

\begin{abstract}
We present two patients who developed visual deterioration due to carbon monoxide poisoning. They were treated with hyperbaric oxygen and recovered not only their vision but also they improved neurological signs and symptoms. We believe that implementation of hyperbaric oxygen, even in a late period of time will be effective in reversing neurological sequelae. Key words: carbon monoxide poisoning, blindness, brain injuries, child, hyperbaric oxygenation.
\end{abstract}

http:/ / dx.doi.org/10.5546/ aap.2020.e480

Cómo citar: Cardoso PC, La Fuente A, Subbotina N, Cargnel EG. Ceguera y trastornos neurológicos por monóxido de carbono: tratamiento efectivo con oxígeno hiperbárico en dos pacientes pediátricos. Arch Argent Pediatr 2020;118(5):e480-e485.

a. Unidad de Toxicología, Hospital de Niños "Ricardo Gutiérrez".

b. Servicio de Neurología, Hospital de Niños "Ricardo Gutiérrez".

c. Centro de Medicina Hiperbárica Buenos Aires.

Argentina.

Correspondencia:

Dra. Patricia C. Cardoso: patriciaceciliacardoso@gmail.com

Financiamiento: Ninguno.

Conflicto de intereses: Ninguno que declarar.

Recibido: 16-12-2019

Aceptado: 16-6-2020

\section{INTRODUCCIÓN}

La intoxicación aguda por inhalación de monóxido de carbono (CO), gas producto de una combustión incompleta y, sobre todo, que forma parte del humo de incendio, puede ocasionar un daño grave en el sistema nervioso central (SNC). Si bien la ceguera puede ser una secuela, existen contados reportes publicados sobre esta en niños y su tratamiento "exitoso" con oxígeno hiperbárico (OHB). ${ }^{1}$ El objetivo de presentar estos dos casos es divulgar esta terapéutica de múltiples sesiones de OHB ante la secuela de ceguera y otros signos neurológicos.

\section{CASO CLÍNICO 1}

Niño de 11 años, previamente sano, que inhaló humo de incendio y fue hallado con pérdida de conocimiento, palidez, piel y narinas tiznadas, en paro cardiorrespiratorio. Unos vecinos realizaron maniobras de resucitación cardiopulmonar. Durante el traslado al hospital zonal, se despertó y respondió a preguntas formuladas. Presentó un episodio de excitación psicomotriz seguido de dificultad en el habla y permaneció irritable.

Al día siguiente, estaba menos irritable, pero sufría cefalea punzante intermitente e intolerancia oral. La tomografía axial computada (TAC) cerebral, el electroencefalograma y el electrocardiograma no mostraban alteraciones patológicas. A las 48 h (después de la intoxicación), comenzó con visión borrosa hasta la ceguera; a las $72 \mathrm{~h}$, presentó mutismo y no respondía a órdenes simples.

Posteriormente a una consulta telefónica a la Unidad de Toxicología, se aceptó la derivación al Hospital de Niños "Ricardo Gutiérrez" a los 5 días del evento. Ingresó con bradicardia, respiración superficial y reflejos abolidos, y recibió asistencia respiratoria mecánica (ARM). Se realizó una resonancia magnética nuclear (RMN) cerebral y el estudio de líquido cefalorraquídeo, sin alteraciones. 
Luego de la extubación electiva a los 8 días, presentaba deterioro neurológico marcado, por lo que, al día siguiente, una vez estabilizado, nuestra Unidad indicó la primera sesión de OHB. Al finalizar, presentó una crisis de llanto, síntoma determinante para decidir continuar el tratamiento con OHB, ya que se consideró de buen pronóstico. Al día siguiente, se observó una

Figura 1. Paciente1. Resonancia magnética nuclear de cerebro, lesiones hiperintensas bilaterales en región parietooccipital

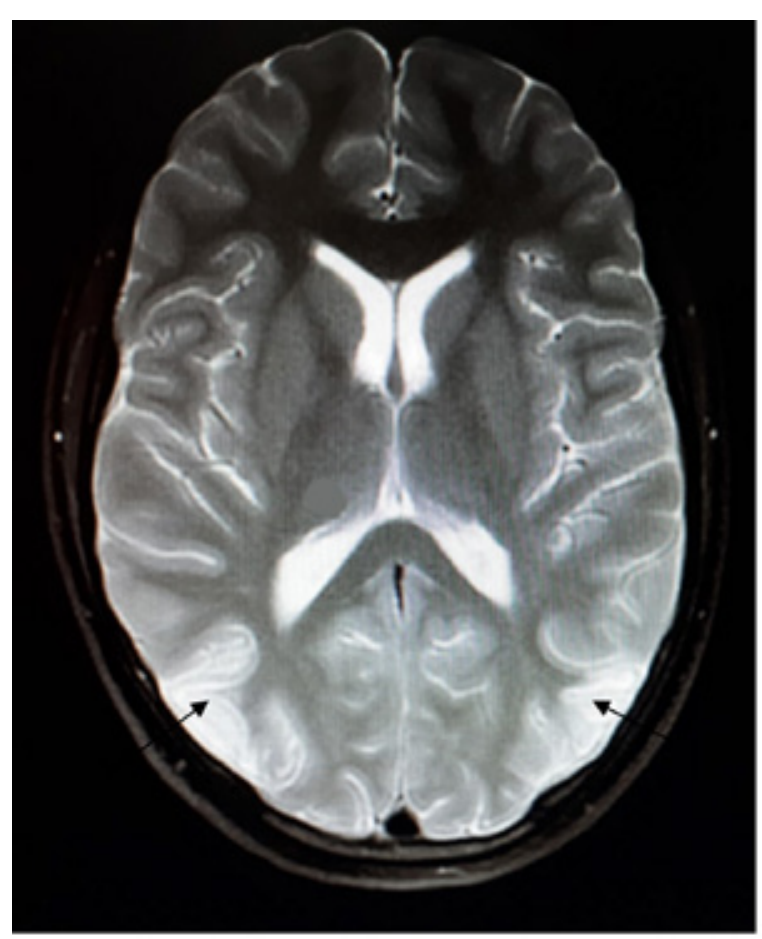

rápida mejoría neurológica con la segunda sesión, lo que reforzó la continuidad terapéutica diaria, de lunes a viernes. La evolución neurológica se detalla en la Tabla 1. La Figura 1 muestra los resultados de la RMN cerebral.

Se realizaron 35 sesiones de $\mathrm{OHB}$ en total. $\mathrm{Al}$ término, recuperó la agudeza visual, con franca evolución positiva de su cuadro neurológico. Se solicitaron estudios por imágenes (RMN con tomografía por emisión de fotón único -single photon emission computed tomography; SPECT, por sus siglas en inglés- de cerebro), que mostraron lesiones secuelares a nivel parietooccipital y frontal, aunque, en la SPECT cerebral con Tc99, con respecto al estudio previo, tuvo franca mejoría de la distribución de la concentración parenquimatosa del trazador, aunque persistió cierto compromiso.

El paciente continuó con rehabilitación motora y apoyo escolar, y logró un buen rendimiento académico. Un año después, presentó una convulsión tónica de minutos de duración, considerada como secundaria al daño secuelar del SNC. Se realizó un electroencefalograma y se observaron descargas de espigas frontales cerebrales (Figura 2), por lo que se comenzó el tratamiento con clobazam.

\section{CASO CLÍNICO 2}

Adolescente de 13 años, previamente sano, expuesto a humo de incendio en la vivienda, durante, aproximadamente, 40 minutos. El hermano mellizo falleció y él fue trasladado al hospital zonal. Ingresó en coma, con cianosis generalizada con insuficiencia ventilatoria y bradicardia extrema. Recibió ARM durante 4 días

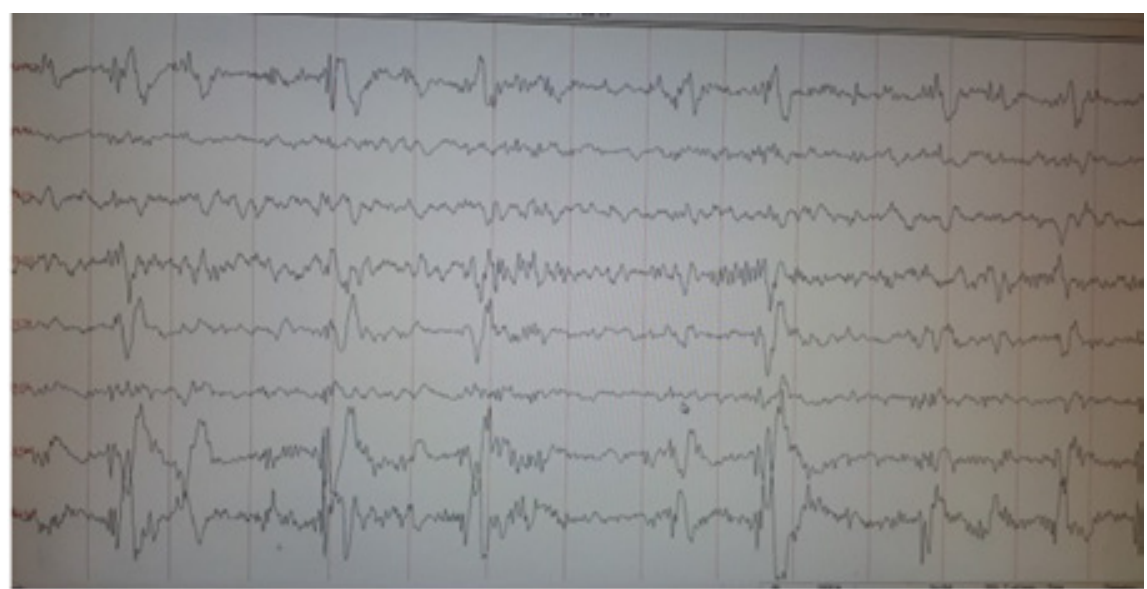


e482 / Arch Argent Pediatr 2020;118(5):e480-e485 / Presentación de casos clínicos

TABLa 1. Caso clínico 1. Evolución del cuadro visual y demás signos neurológicos, y estudios realizados antes, durante y después del tratamiento con oxígeno hiperbárico

\begin{tabular}{|c|c|c|}
\hline Días pos intoxicación por $\mathrm{CO}$ & Evolución neurológica & Estudios \\
\hline $\begin{array}{l}8 \text { días } \\
\text { (antes del tratamiento con } \mathrm{OHB} \text { ). }\end{array}$ & $\begin{array}{l}\text { Respondía preguntas simples con } \\
\text { monosílabos y dificultad. } \\
\text { Alteración visual, tono muscular conservado, } \\
\text { aunque sin movilizarse y ROT aumentados. }\end{array}$ & $\begin{array}{l}\text { RMN cerebral y estudio de líquido } \\
\text { cefalorraquídeo sin alteraciones } \\
\text { patológicas. La RMN cerebral era } \\
\text { compatible con isquemia (Figura 1). } \\
\text { Electroencefalograma: actividad } \\
\text { delta disminuida en forma } \\
\text { generalizada, sin descargas ni } \\
\text { paroxismos locales. }\end{array}$ \\
\hline
\end{tabular}

\begin{tabular}{ll}
\hline 9 días (sesión 1). & Presentó una crisis de llanto. \\
\hline 10 días (sesión 2). & Habla enlentecida, coherente; visión de bulto \\
en forma intermitente; reconocía movimientos, \\
formas y colores, pero no seguía objetos \\
con la mirada. \\
FO derecho: papila de bordes levemente difusos; \\
izquierdo: borde nasal con leve borramiento.
\end{tabular}

\begin{tabular}{lll}
\hline 11 días. & Comenzó a deambular con ayuda e & Electroencefalograma: moderada \\
& ingirió líquidos sin dificultad. & desorganización sin foco \\
& A los 16 días, deambulaba sin ayuda, & paroxístico.
\end{tabular}
con pasos cortos y temblor distal; dificultad en la coordinación, habla enlentecida, pero podía responder con sentido del humor.

\begin{tabular}{|c|c|c|}
\hline 23 días (sesión 14). & $\begin{array}{l}\text { Recordó el día de la intoxicación; } \\
\text { conteo de hasta } 5 \text { con una mano. }\end{array}$ & $\begin{array}{l}\text { Tomografía por emisión de un solo } \\
\text { fotón cerebral, con Tc99 (SPECT } \\
\text { de cerebro con Tc99): extensa y muy } \\
\text { significativa disminución de } \\
\text { la concentración de tecnecio } \\
\text { bilateral en ambas regiones } \\
\text { temporoparietales, que, en algunas } \\
\text { áreas, llegaba a la ausencia de } \\
\text { concentración significativa } \\
\text { detectable e involucraba los giros } \\
\text { temporales superiores, medios e } \\
\text { inferiores y los lóbulos parietales } \\
\text { superior e inferior. Presentación } \\
\text { similar sobre ambos hemisferios } \\
\text { corticales frontales, algo más } \\
\text { extensa y pronunciada del lado } \\
\text { izquierdo, en territorio del giro } \\
\text { frontal medio y giro precentral. }\end{array}$ \\
\hline
\end{tabular}

\begin{tabular}{|c|c|c|}
\hline 30 días. & $\begin{array}{l}\text { Agudeza visual con evolución favorable, } \\
\text { OD y OI: 0,6; FO: normal; visión cromática } \\
\text { conservada, alteración en la percepción } \\
\text { de profundidad. } \\
\text { Potenciales evocados visuales y auditivos normales. } \\
\text { Podía andar en patineta de } 2 \text { ruedas. }\end{array}$ & \\
\hline 40 días. & $\begin{array}{l}\text { Mejoría de la motricidad fina: podía manipular } \\
\text { un cierre y atarse los cordones. }\end{array}$ & \\
\hline 60 días (35 sesiones). & $\begin{array}{l}\text { Recuperó totalmente la agudeza visual. } \\
\text { Leve hiperreflexia patelar izquierda, con el resto } \\
\text { del examen físico neurológico normal y } \\
\text { función mnésica comprometida; podía leer } \\
\text { pequeños fragmentos y realizar grafismos } \\
\text { con ayuda. }\end{array}$ & $\begin{array}{l}\text { Electroencefalograma: trazado de } \\
\text { RMN con SPECT de cerebro: } \\
\text { transición y somnolencia normal. } \\
\text { lesiones secuelares a nivel } \\
\text { parietooccipital y frontal. } \\
\text { SPECT cerebral con Tc99, con franca } \\
\text { mejoría de la distribución de la } \\
\text { concentración parenquimatosa } \\
\text { cerebral -del trazador-; persistía el } \\
\text { compromiso a nivel parietal } \\
\text { bilateral y frontal, con mejoría en } \\
\text { las áreas temporales. }\end{array}$ \\
\hline 12 meses. & $\begin{array}{l}\text { Convulsión tónica de minutos de duración, } \\
\text { asumida como secundaria al daño secuelar } \\
\text { a nivel del SNC. }\end{array}$ & $\begin{array}{l}\text { Electroencefalograma: descargas de } \\
\text { espigas frontales cerebrales } \\
\text { (Figura 2). }\end{array}$ \\
\hline
\end{tabular}

CO: monóxido de carbono; OHB: oxígeno hiperbárico; ROT: reflejos osteotendinosos; RMN: resonancia magnética nuclear;

FO: fondo de ojos; OD: ojo derecho; OI: ojo izquierdo; SPECT: tomografía por emisión de fotón único; SNC: sistema nervioso central. 
y, luego de la extubación, permaneció irritable y desorientado. Su estado de conciencia mejoró posteriormente, con dificultad en la marcha de predominio izquierdo, disminución en la fuerza muscular, temblor distal en los miembros superiores y visión de bulto (fondo de ojo -FO- normal) y una RMN cerebral alterada.

A los 29 días, la médica de cabecera lo derivó a nuestra Unidad de Toxicología, ya que presentaba un cuadro secuelar neurológico marcado. Se indicó internación y OHB. Luego de la primera sesión, ante una rápida mejoría de su visión,

TABLA 2. Caso clínico 2. Evolución del cuadro visual y demás signos neurológicos, y estudios realizados antes, durante y después del tratamiento con oxígeno hiperbárico

\begin{tabular}{|c|c|c|}
\hline Días pos intoxicación por $\mathrm{CO}$ & Evolución neurológica & Estudios \\
\hline $\begin{array}{l}29 \text { días } \\
\text { (antes del tratamiento con } \mathrm{OHB} \text { ). }\end{array}$ & $\begin{array}{l}\text { Hablaba sin dificultad; leve hemiparesia izquierda, } \\
\text { deambulaba con ligera lateralización hacia la derecha } \\
\text { y temblor fino distal de los miembros superiores } \\
\text { de predominio izquierdo, Babinski positivo y } \\
\text { leve rueda dentada. Presentaba trastornos de memoria, } \\
\text { visión de bulto (agudeza visual, OD: } 0,1 \text {; OI: } 0,1 \text { ). }\end{array}$ & $\begin{array}{l}\text { Electroencefalograma: } \\
\text { desorganizado, sin focos ni } \\
\text { paroxismos. } \\
\text { TAC cerebral: no mostró lesiones. } \\
\text { RMN cerebral: hiperintensidad en } \\
\text { secuencia flair en surcos corticales de } \\
\text { la convexidad a nivel } \\
\text { occipitoparietal bilateral y a nivel } \\
\text { frontal del lado derecho. }\end{array}$ \\
\hline
\end{tabular}

29 días (sesión 1). $\quad$ Luego de la primera sesión, el paciente refirió ver mejor

"sin chocarse" con otras personas. Al día siguiente

(sesión 2), logró leer "letras grandes" de un cartel a 2 m.

\begin{tabular}{ll}
\hline 36 días (sesión 5). & Agudeza visual en OD: 0,6; OI: 0,5. \\
\hline 39 días. & Deambuló con mayor estabilidad y mejoría de \\
& la hemiparesia izquierda, y conservó habilidades cognitivas, \\
& lenguaje y memoria. \\
& Habilidades de integración visomotriz notablemente \\
& descendidas, condicionadas por la dificultad \\
& en la motricidad fina. \\
\hline
\end{tabular}

42 días. $\quad$ Marcha levemente inestable, fuerza muscular conservada, sin Babinski ni rueda dentada, con ROT conservados, y persistió un leve temblor distal.

60 días. $\quad$ Agudeza visual de ambos ojos de 10/10 con FO normal. Potenciales evocados visuales, auditivos y somatosensitivos dentro de los parámetros normales.

Electroencefalograma: trazado de vigilia y sueño normal. Electromiograma: compromiso radiculomotor parcialmente compensado por reinervación de colaterales axónicas y sin actividad denervatoria a nivel del miotoma L5 izquierdo.

\begin{tabular}{lll}
\hline 70 días (sesión 28). & Escaso temblor distal. & \\
\hline 90 días (sesión 30). & $\begin{array}{l}\text { Mejoró la escritura y logró atarse los cordones } \\
\text { de los zapatos. }\end{array}$ & $\begin{array}{l}\text { RMN cerebral: áreas secuelares a } \\
\text { nivel cortical frontal derecho y } \\
\text { parietooccipital posterior, imagen } \\
\text { focal secuelar en globo pálido } \\
\text { derecho y putamen izquierdo (Figura 3). }\end{array}$ \\
& $\begin{array}{l}\text { Espectroscopía: en las lesiones } \\
\text { descritas, existía reducción de NAA, } \\
\text { presencia de mioinositol y lactato }\end{array}$ \\
& compatibles con áreas secuelares \\
& cavitadas asociadas a gliosis (Figura 4). \\
\hline
\end{tabular}


se decidió continuar con el tratamiento de OHB diariamente, de lunes a viernes, mientras continuara su progreso.

La evolución neurológica se detalla en la Tabla 2. Luego de 30 sesiones y habiendo transcurrido 90 días, ante la mejoría clínica continua, se indicaron 5 sesiones más. Se efectuó el estudio de RMN (Figura 3.a). Además, en este caso, se tuvo en cuenta la espectroscopía por resonancia magnética (ERM) (Figura 3.b). El paciente recibió un total de 40 sesiones de OHB $\mathrm{y}$, a los 19 meses del episodio, no se observaban temblores, con mejoría paulatina de la escritura.

\section{DISCUSIÓN}

Luego de la intoxicación aguda grave por $\mathrm{CO}$, pueden presentarse signos neurológicos, sean como secuela posepisodio o aparecer luego de una aparente normalidad como "síndrome neurológico tardío" (SNT), con un período de ventana entre los 2 y los 40 días, y una mediana de aparición a los 20 días. Este último se debería a un daño cerebral que no solo se asocia a hipoxia, sino también a otros mecanismos que provocan la aparición de radicales libres, con desmielinización. De acuerdo con varios autores, se puede observar reversión del síndrome en, aproximadamente, el $70 \%$ de los pacientes y puede llevar de uno a dos años. ${ }^{2}$

Figura 3.a. Paciente 2. Resonancia magnética nuclear cerebral: hiperintensidad de globo pálido, putamen y cortical parietooccipital

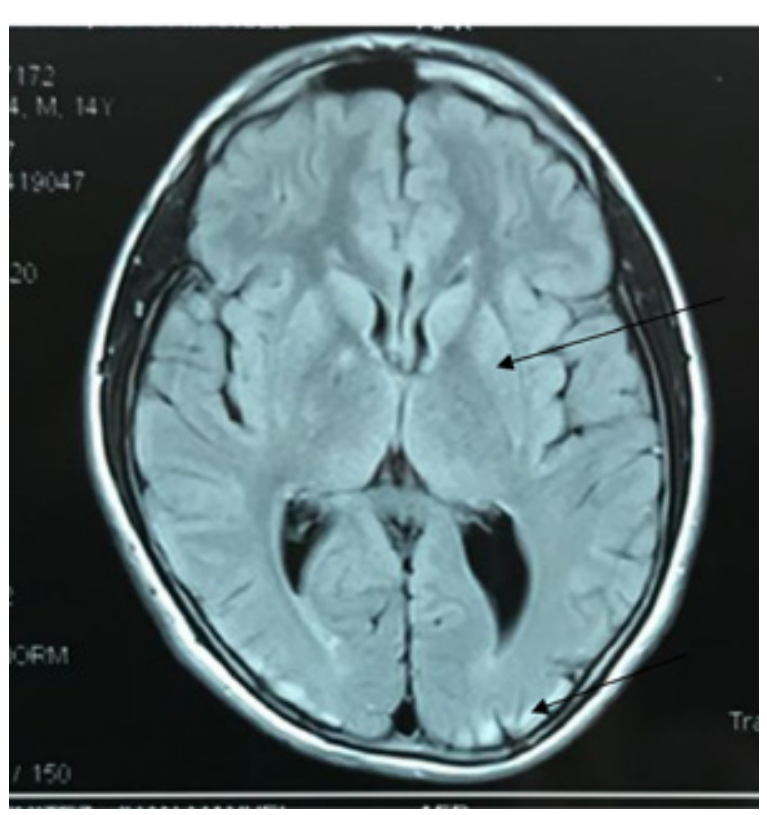

Los mecanismos de acción postulados para el tratamiento con OHB son varios (aceleración de eliminación del CO, mejoría del metabolismo oxidativo mitocondrial, inhibición de la peroxidación lipídica, inhibición de la adherencia de los leucocitos a la microvasculatura dañada, atenuación de la disfunción neurológica inmunomediada), pero algunos autores proponen otro: si bien algunas neuronas se encuentran dañadas e, irreversiblemente, llegan a la necrosis, las células que se hallan cercanas, menos afectadas por la hipoxia e inflamación, formarían una zona de penumbra isquémica. Estas neuronas serían disfuncionales pero aún viables, y pueden reactivarse con $\mathrm{OHB}^{3}$

En cuanto al diagnóstico de estos pacientes, a pesar de no realizarse la determinación de carboxihemoglobina $(\mathrm{COHb})$ al inicio, se consideraron las secuelas por $\mathrm{CO}$ ante la clínica y fuente (CO siempre presente en el humo de incendio, aunque otros compuestos lo puedan acompañar). ${ }^{4}$ Ninguno presentaba secuelas respiratorias.

En el caso clínico 1, el paciente manifestaba pérdida total de la visión a las 48 horas como SNT. En el caso clínico 2, era más difícil determinar el inicio del trastorno visual, debido a que se encontraba sedado con ARM desde un principio.

Se decidió realizar tratamiento con OHB (protocolo: 2,4 atmósferas absolutas -ATAdurante 60 minutos por sesión), a pesar de haber transcurrido varios días desde la intoxicación, en vista de algunas publicaciones que demostraban un beneficio en la amaurosis,, 5 aunque no existiera consenso internacional, ${ }^{7,8}$ Las primeras

FIgURA 3.b. Paciente 2. Resonancia magnética nuclear cerebral con espectroscopía con reducción de N-acetil aspartato y pico de lactato compatible con gliosis

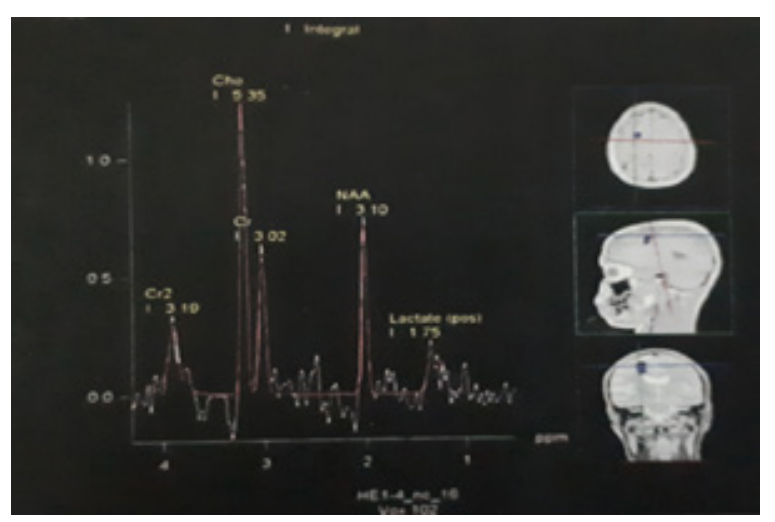


sesiones se realizaron durante la internación en nuestro Hospital; se trasladaron al Centro de Medicina Hiperbárica y luego en forma ambulatoria. Posteriormente a la reversión total del déficit visual y ante otros signos neurológicos que continuaban mejorando, se resolvió proseguir con las sesiones mientras se observara dicha mejoría.

Con respecto a los estudios por imágenes, especialmente la TAC y la RMN cerebral, pueden no observarse lesiones durante los primeros días, por lo que no constituyen una guía adecuada para evaluar la aparición del SNT. Cuando aparecen imágenes, los reportes más frecuentes refieren necrosis del globo pálido y desmielinización de la sustancia blanca cerebral, con predominio del centrum semioval y la sustancia blanca periventricular, pero puede existir compromiso de otras estructuras. ${ }^{9,10}$

En el caso del segundo paciente, para continuar con el tratamiento durante las últimas sesiones, además de la mejoría clínica, se tomó en cuenta la espectroscopía y la tractografía por resonancia magnética (ERM) cerebral, que permitía observar cambios metabólicos en regiones específicas del cerebro dañado. La disminución de $\mathrm{N}$-acetil aspartato traduce una pérdida o degeneración de neuronas, y la presencia de lactato, una glicólisis anaerobia bajo condiciones de isquemia o hipoxia. El lactato comienza a aparecer entre 1 y 2 meses luego de la intoxicación en los pacientes con síntomas crónicos; es un marcador de daño cerebral irreversible, aunque algunos puedan mostrar reversibilidad. El mioinositol se incrementa en las enfermedades desmielinizantes. En el caso del paciente, se decidió agregar nuevas sesiones, y continuó con una evolución positiva., ${ }^{2,10-12}$ Luego de varias sesiones de OHB y de observar una mejoría del cuadro neurológico, se implementó la rehabilitación psicomotriz y psicoterapéutica en ambos pacientes.

Se considera que la reversión completa de la ceguera se debe al tratamiento con $\mathrm{OHB}$ y no a la sola evolución espontánea, debido a que esta coincide con el período de ventana en que, generalmente, se describe la aparición de nuevos signos neurológicos, como SNT, y no su resolución. Al observar la reversión, que incluye también signos neurológicos distintos a la ceguera en un corto período, y tomando en cuenta la gravedad de estos, se aconseja el tratamiento con OHB lo más precozmente posible, sobre todo, si existe sintomatología neurológica y cardíaca. Cuando la decisión de la aplicación de OHB es más tardía, se estima necesaria la evaluación de un especialista en Toxicología.

\section{REFERENCIAS}

1. Katafuchi Y, Nishimi T, Yamaguchi Y, Matsuishi T, et al. Cortical Blindness in Acute Carbon Monoxide Poisoning. Brain Dev. 1985;7(5):516-9.

2. Varrassi M, Di Sibio A, Gianneramo C, Perri M, et al. Advanced neuroimaging of carbon monoxide poisoning. Neuroradiol J. 2017;30(5):461-9.

3. Nemoto EM, Betterman K. Basic physiology of hyperbaric oxygen in brain. Neurol Res. 2007;29(2):116-26.

4. Cardoso P, Alonso M, Cargnel E. Intoxicación por gases. En Neira P, Farías J, Monteverde E. Manual de emergencias pediátricas. Rosario: Corpus; 2011:667-76.

5. Ersanli D, Yildiz S, Togrol E, Ay H, et al. Visual loss as a late complication of carbon monoxide poisoning and its successful treatment with hyperbaric oxygen therapy. Swiss Med Wkly. 2004;134(43-44):650-5.

6. SenolM, YildizS, ErsanliD, Uzun G, etal. Carbon monoxideinduced cortical visual loss: treatment with hyperbaric oxygen four years later. Med Princ Pract. 2009;18(1):67-9.

7. Mathieu D, Marroni A, Kot J. Tenth European Consensus Conference on Hyperbaric Medicine: recommendations for accepted and non-accepted clinical indications and practice of hyperbaric oxygen treatment. Diving Hyperb Med. 2017;47(1):24-32.

8. Lin $\mathrm{CH}$, Su $\mathrm{WH}$, Chen $\mathrm{YC}$, Feng $\mathrm{PH}$, et al. Treatment With Normobaric or Hyperbaric Oxygen and Its Effect on Neuropsychometric Dysfunction After Carbon Monoxide Poisoning: A Systematic Review and Meta-Analysis of Randomized Controlled Trials. Medicine (Baltimore). 2018;97(39):e12456.

9. Hopkins RO, Woon FL. Neuroimaging, cognitive, and neurobehavioral outcomes following carbon monoxide poisoning. Behav Cogn Neurosci Rev. 2006;5(3):141-55.

10. O'Donnell P, Buxton PJ, Pitkin A, Jarvis LJ. The magnetic resonanceimaging appearances of the brain in acute carbon monoxide poisoning. Clin Radiol. 2000;55(4):273-80.

11. Beppu T. The role of MR imaging in assessment of brain damage from carbon monoxide poisoning: a review of the literature. AJNR Am J Neuroradiol. 2014;35(4):625-31.

12. Terajima $K$, Igarashi $H$, Hirose $M$, Matsuzawa $H$, et al. Serial assessment of delayed encephalopathy after carbon monoxide poisoning using magnetic resonance spectroscopy and diffusion tensor imaging on 3.0T system. Eur Neurol. 2008;59(1-2):55-61. 